\title{
Autologous cell replacement: a noninvasive Al approach to clinical release testing
}

\author{
Budd A. Tucker, Robert F. Mullins, and Edwin M. Stone \\ Institute for Vision Research, Department of Ophthalmology and Visual Science, Carver College of Medicine, The University of lowa, lowa City, lowa, USA.
}

The advent of human induced pluripotent stem cells (iPSCs) provided a means for avoiding ethical concerns associated with the use of cells isolated from human embryos. The number of labs now using iPSCs to generate photoreceptor, retinal pigmented epithelial (RPE), and-more recently-choroidal endothelial cells has grown exponentially. However, for autologous cell replacement to be effective, manufacturing strategies will need to change. Many tasks carried out by hand will need simplifying and automating. In this issue of the $J C I$, Schaub and colleagues combined quantitative bright-field microscopy and artificial intelligence (deep neural networks and traditional machine learning) to noninvasively monitor iPSC-derived graft maturation, predict donor cell identity, and evaluate graft function prior to transplantation. This approach allowed the authors to preemptively identify and remove abnormal grafts. Notably, the method is (a) transferable, (b) cost and time effective, (c) high throughput, and (d) useful for primary product validation.

\section{Solid organ transplant}

Although the concept of tissue replacement as a means of restoring normal bodily function has existed for millennia, the first successful solid organ transplant was not performed until 1954, when Murray and colleagues used a patient's identical twin as a kidney donor to circumvent immunemediated rejection $(1,2)$. Following Murray's original report, a push was made to develop effective antirejection drugs, which were designed to prevent the host immune system from destroying the newly transplanted tissue $(3,4)$. HLA-based tissue typing was also developed to identify organ donors that more closely matched the recipient $(5,6)$. Since these landmark studies, solid organ transplantation has become mainstream, and countless years of productive human life have been saved (7).

Unlike the loss of function of the kidney, loss of function following death of CNS neurons is usually permanent. Attempts to restore vision by transplanting whole eyes, which requires CNS axons from the donor tissue to extend into and make new synaptic connections with the host midbrain, have been unsuccessful. Once cut, CNS axons are unable to navigate the long distances required to reinnervate their original target and restore normal function. The inability of mature CNS neurons to regenerate is largely the result of an inhospitable extracellular environment, which contains inhibitory myelin-associated (8) and injuryinduced gliotic proteins (9). To address the fairly insurmountable limitations of wholeeye transplant, most modern-day researchers focus on replacing lost cell types with stem cell-based treatments.

\section{The human retina}

The human retina is a highly specialized tissue that overlays the inner surface of the

Related Article: p. 1010

Conflict of interest: The authors have declared that no conflict of interest exists.

Copyright: @ 2020, American Society for Clinical Investigation.

Reference information: / Clin Invest. 2020;130(2):608-611. https://doi.org/10.1172/JCl133821.

posterior portion of the eye. It consists of the inner neural retina and an underlying monolayer of non-neural retinal pigmented epithelial (RPE) cells (Figure 1A). The neural retina, like the cerebral cortex, is a laminated structure made up of a variety of neural subtypes. From a simplistic flow-of-information perspective, light is detected by photoreceptor cells in the outermost layer adjacent to the RPE layer, where a series of biochemical actions result in an electrochemical response that is propagated to the brain via the overlying bipolar interneurons and retinal ganglion cells (Figure 1A). Among its many functions, the $\mathrm{RPE}$ layer is responsible for recycling of the vitamin A-based chromophore that is needed by the photoreceptor cells to detect light. Beneath the RPE layer is a specialized vascular bed known as the choroid (Figure 1A). This layer is primarily responsible for providing nutrients to and removing waste from the overlying RPE and photoreceptor cells. With the exception of death of the bipolar interneurons, death of each of these cell types has been associated with major forms of incurable blindness.

\section{Clinical application for the ideal cell type}

The field of stem cell biology, and in particular retinal cell replacement, has grown dramatically over the past two decades. For retinal degenerative disorders such as retinitis pigmentosa (RP) and age-related macular degeneration (AMD), the majority of the cell replacement effort has gone into (a) selection of the appropriate donor cell type, (b) derivation of effective differentiation protocols, and (c) development of appropriate cell delivery methods. Since the turn of the century, numerous studies have been performed using a variety of donor cell types for retinal cell replacement, ranging from pluripotent embryonic stem cells to fate-restricted neural progenitor cells isolated from the hippocampus. Following the isolation of pluripotent human embryonic stem cells by Thomson 
A

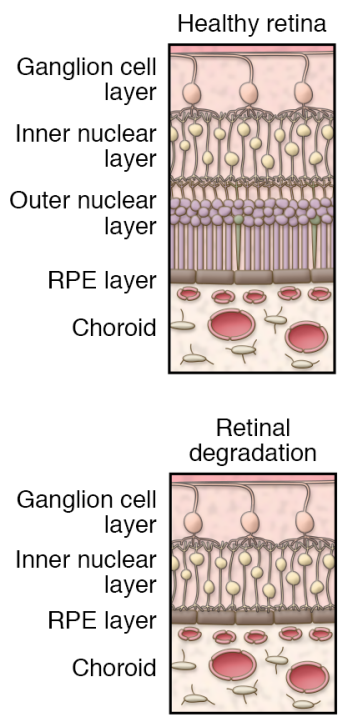

B

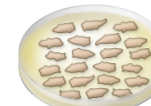

Dermal fibroblast isolation

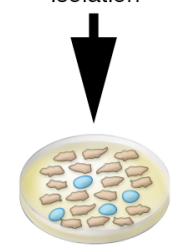

iSPC generation and expansion

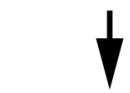

CRISPR correction

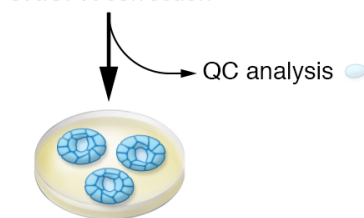

Differentiation to retinal organoids
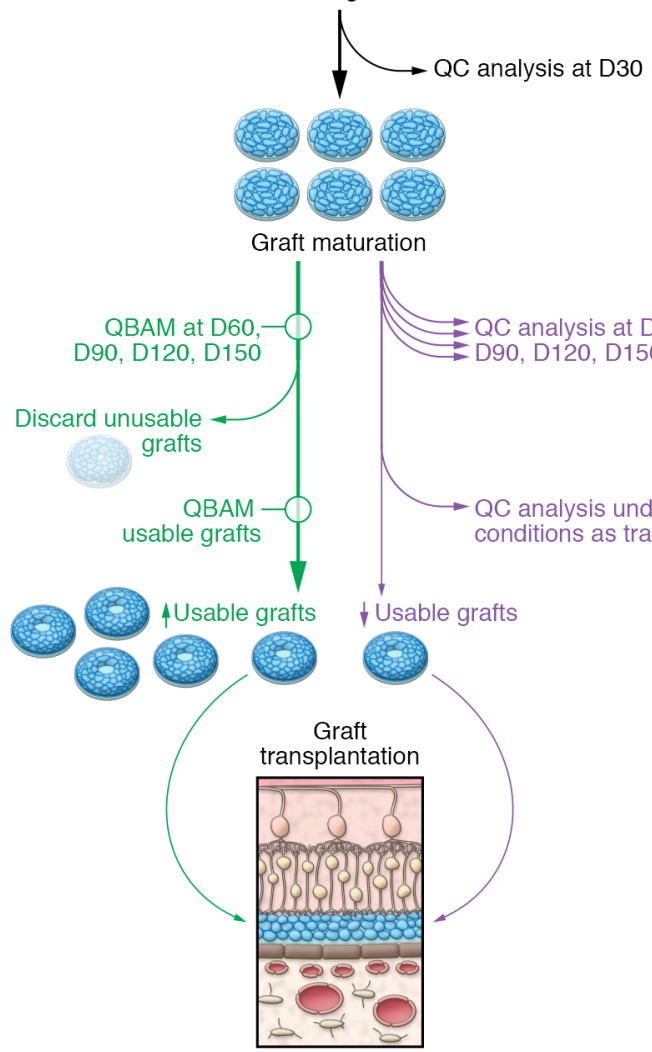

et al. in 1998 (10), many researchers chose to focus their attention on developing differentiation protocols suitable for generating both photoreceptor cells and RPE cells. In 2004, Haruta et al. published one of the earliest studies demonstrating the generation of RPE cells from primate embryonic stem cells (ESCs), which, when transplanted into the subretinal space of rats with a primary
RPE cell defect, prevented retinal degeneration (11). Just two years later, Lamba and colleagues demonstrated that by using a stepwise differentiation protocol they could generate photoreceptor cells from human ESCs that were capable of integrating with a dystrophic host retina (12). With what would appear to be the ideal cell type and appropriate differentiation protocols
Figure 1. Schematic depiction of a hypothetical clinical manufacturing pipeline for an autologous retinal cell graft . (A) Anatomy of normal human retina and retinal anatomy of a patient with end-stage inherited retinal degenerative blindness with loss of the photoreceptor cell layer. (B) Graft implant process. First, dermal fibroblast cell isolation and expansion. Second, iPSC generation and clonal expansion. Third, CRISPR correction of the patient's diseasecausing genetic mutations. Fourth, QC analysis of CRISPR-corrected patient-derived iPSCs, which includes confirmation of pluripotency, retinal differentiation potential, normal karyotype, and genetic correction in the absence of off-target editing. Fifth, initiation of $3 D$ retinal differentiation. Sixth, QC analysis to demonstrate retinal progenitor cell differentiation at day 30 . Seventh, retinal organoids are dissociated and isolated retinal progenitor cells are loaded onto retinal cell delivery scaffolds and matured. Eighth, production pipeline of retinal cell grafts harvested at $60,90,120$, and 150 days after differentiation to confirm normal maturation, sterility, potency, and identity (shown in purple). Production pipeline, using QBAM retinal cell grafts, can be evaluated at the same intervals without the need to harvest (shown in green). Unusable grafts can be discarded. Ninth, subretinal transplantation of one of the remaining retinal cell grafts. By using the QBAM-based live release testing strategy outlined in green, the number of grafts required for each patient is significantly reduced. Likewise, as compared with the traditional "go-no-go" pipeline depicted in purple, by using a QBAM-based approach, unusable grafts can be discarded during maturation, and the best graft available can be selected for transplantation (17). iPSC, induced pluripotent stem cell; QBAM, quantitative brightfield microscopy; QC, quality control. in hand, the remaining hurdle for effective clinical application has shifted to identifying the best method of delivery. Studies performed in animals have shown that injection of cells into the subretinal space as an unorganized suspension, regardless of cell type, results in mass cell death and poor integration with host tissue (13). For these reasons several labs have chosen to 
develop cell delivery scaffolds designed to carry the cells as an intact graft, which is proving effective (14).

The field has begun using stem cells in clinical trials to replace RPE cells in patients with $\operatorname{AMD}(15,16)$. As a result, investigators are identifying aspects of traditional clinical-grade manufacturing and product release testing that will need to be improved before mass implementation. This is especially true for autologous iPSCbased approaches, in which each product is specifically manufactured for a single individual. A typical iPSC production pipeline begins with the identification of the disease state and the specific cell type required for treatment (e.g., photoreceptor cells for RP, Figure 1B). The next step is to obtain somatic cells from the donor, typically dermal fibroblasts or bloodderived mononuclear cells, and generate iPSCs (Figure 1B). Once generated, if the disease being treated is Mendelian, scientists may need to correct the patient's disease-causing genetic mutations (CRISPR correction, Figure 1B). Following correction, cells will need validating (QC analysis, Figure 1B). This process typically involves karyotyping, evaluation of pluripotency and differentiation potential, and if genetically corrected, confirmation that the line is free from deleterious off-target editing events. Once fully validated, cells must go through a series of differentiation steps before being loaded into a cell delivery scaffold, where they are matured for a predetermined period before release to the clinic for transplantation into the patient's subretinal space (Figure 1B). At a variety of stages throughout this process, quality checks are made to determine whether the product is developing properly. In most cases, these quality checks will require terminal evaluation, where a sample that is presumed identical to the product destined to be transplanted is taken and a series of tests are performed.

\section{Clinical release criteria}

In an elegant study published in this issue of the JCI, Schaub and colleagues report a strategy that combines the use of QBAM and artificial intelligence (AI; namely, deep neural networks and traditional machine learning) to noninvasively monitor graft maturation, predict donor cell identity, and evaluate graft function prior to trans- plantation (17). The authors hypothesized that the physical appearance of pluripotent stem cell-derived progeny is highly correlated with and predictive of cellular function. To validate their system, several iPSC lines from patients with either ocular albinism or AMD, as well as from normal nondiseased individuals, were generated. These cell lines were differentiated into RPE cells, which were seeded onto porous Transwell inserts for maturation. Drugs that are known to induce or inhibit the RPE maturation process were also included. By using visual features extracted from the QBAM images obtained during differentiation and maturation, the authors were able to use deep neural networks and traditional machine learning to accurately predict transepithelial resistance and polarized VEGF secretion, both of which are features of RPE cell function and indicative of graft maturation. Importantly, by including cell lines generated from patients with ocular albinism, which lack pigment, the authors were able to evaluate QBAM sensitivity. Similarly, including clinical-grade lines from patients with AMD allowed the authors to demonstrate that their platform was robust across clinically relevant sample sets. This approach allowed the authors to preemptively identify and remove abnormal grafts, keeping only products that met their stringent clinical release criteria (e.g., Figure 1B, green pathway). In addition, the authors were able to predict the lineage of RPE cell grafts with outstanding accuracy; specifically, they were able to accurately predict whether RPE cells came from the same donor $85 \%$ of the time (17).

This approach has several major advantages over current "go-no-go" quality and release testing paradigms (i.e., Figure $1 \mathrm{~B}$, purple pathway). It is (a) transferable, (b) cost- and time effective, (c) high throughput, and (d) useful for primary product validation.

Transferable. One of the greatest strengths of the described approach is that it uses existing nonproprietary technology. Specifically, researchers can implement the QBAM system using any standard brightfield microscope and replicate the deep neural network/traditional machine learning approaches described.

Cost-and time effective. By implementing the progressive nonterminal quality evalua- tion protocol described, one can effectively reduce the number of grafts required for release testing. By decreasing the number of grafts required for each patient, one can increase the number of patient samples for parallel processing, drastically reducing the per patient product cost.

High throughput. By combining the QBAM/AI system described (17) with automatic imaging and robotic cell culture platforms, one could readily envision parallel processing-based scale-up of cell differentiation and graft validation. For autologous cell replacement approaches, which are not well suited to traditional bulk scale-up, technologies such as these will be key.

Useful for primary product validation. Unlike terminal assays, the described approach is completely noninvasive and available to validate the function and identity of the actual product throughout the manufacturing process until the point of use. This effectively mitigates concerns that the product being delivered could be inferior to the samples that were taken during manufacturing quality checks and product release.

\section{Future direction and conclusions}

Before the discovery that pluripotent stem cells could be generated from human somatic cells via delivery of just four transgenes, autologous cell replacement for the treatment of neurodegenerative disorders, such as RP, was impossible. In the preiPSC era, where scale-up simply meant bulk production of a single nonimmunologically matched product for all patients, traditional manufacturing strategies were sufficient. Future manufacturing strategies will need to focus on scale-out (parallel production) rather than scale-up (bulk production). The QBAM-based approach Schaub and colleagues (17) described is an excellent step toward parallel (scale-out) production that reduces the process complexity and toward automation of tasks typically carried out by hand. The methods described are robust, are transferable to other labs and organ systems, and provide tools to reduce production costs associated with replicate samples and required worker hours. As an additional advantage, the strategy presented allows for meaningful evaluation of the actual product that will be delivered to a patient. 
Expanding this approach to include confocal or similar imaging systems that can evaluate cells in the $z$ dimension is desirable. Because restoring high-acuity vision to patients with advanced AMD may require multilayered 3D tissue constructs containing several cell types (e.g., photoreceptor, RPE, and choroidal endothelial cells), the ability to apply a QBAM/AI approach (17) to evaluate $3 \mathrm{D}$ grafts during production will be very valuable.

Address correspondence to: Budd A. Tucker, 375 Newton Road, Iowa City, Iowa 52242, USA. Phone: 319.353.4488; Email: budd-tucker@uiowa.edu.

1. Merrill JP, Murray JE, Harrison JH, Guild WR. Successful homotransplantation of the human kidney between identical twins. J Am Med Assoc. 1956;160(4):277-282.

2. Harrison JH, Merrill JP, Murray JE. Renal homotransplantation in identical twins. Surg Forum.
1956;6:432-436.

3. Schwartz R, Dameshek W. The effects of 6mercaptopurine on homograft reactions. J Clin Invest. 1960;39:952-958.

4. Starzl TE, Marchioro TL, Waddell WR. The reversal of rejection in human renal homografts with subsequent development of homograft tolerance. Surg Gynecol Obstet. 1963;117:385-395.

5. Thorsby E. A short history of HLA. Tissue Antigens. 2009;74(2):101-116.

6. Morris AB, Sullivan HC, Krummey SM, Gebel HM, Bray RA. Virtual versus physical crossmatching in the modern era. HLA. 2019;94(6):471-481.

7. Rana A, et al. Survival benefit of solid-organ transplant in the United States. JAMA Surg. 2015;150(3):252-259.

8. Schwab ME, Bandtlow CE. Neurobiology. Inhibitory influences. Nature. 1994;371(6499):658-659.

9. Morgenstern DA, Asher RA, Fawcett JW. Chondroitin sulphate proteoglycans in the CNS injury response. Prog Brain Res. 2002;137:313-332.

10. Thomson JA, et al. Embryonic stem cell lines derived from human blastocysts. Science. 1998;282(5391):1145-1147.

11. Haruta $\mathrm{M}$, et al. In vitro and in vivo characterization of pigment epithelial cells differentiated from primate embryonic stem cells. Invest Ophthalmol Vis Sci. 2004;45(3):1020-1025.

12. Lamba DA, Karl MO, Ware CB, Reh TA. Efficient generation of retinal progenitor cells from human embryonic stem cells. Proc Natl Acad Sci US A. 2006;103(34):12769-12774.

13. Tomita M, Lavik E, Klassen H, Zahir T, Langer $\mathrm{R}$, Young MJ. Biodegradable polymer composite grafts promote the survival and differentiation of retinal progenitor cells. Stem Cells. 2005;23(10):1579-1588.

14. Sharma R, et al. Clinical-grade stem cell-derived retinal pigment epithelium patch rescues retinal degeneration in rodents and pigs. Sci Transl Med. 2019;11(475):eaat5580.

15. Kashani AH, et al. A bioengineered retinal pigment epithelial monolayer for advanced, dry age-related macular degeneration. Sci Transl Med. 2018;10(435):eaao4097.

16. Mandai M, et al. Autologous induced stem-cellderived retinal cells for macular degeneration. N Engl J Med. 2017;376(11):1038-1046.

17. Schaub NJ, et al. Deep learning predicts function of live retinal pigment epithelium from quantitative microscopy. J Clin Invest. 2020;130(2):1010-1023. 